\title{
Video Article \\ Extraction of Aqueous Metabolites from Cultured Adherent Cells for Metabolomic Analysis by Capillary Electrophoresis-Mass Spectrometry
}

\author{
Ami Maruyama ${ }^{1,2}$, Kenjiro Kami ${ }^{3}$, Kazunori Sasaki ${ }^{3}$, Hajime Sato ${ }^{3}$, Yuzo Sato ${ }^{1,2}$, Katsuya Tsuchihara ${ }^{4}$, Hideki Makinoshima ${ }^{1,2,4}$ \\ ${ }^{1}$ Shonai Regional Industry Promotion Center \\ ${ }^{2}$ Tsuruoka Metabolomics Laboratory, National Cancer Center \\ ${ }^{3}$ Human Metabolome Technologies, Inc \\ ${ }^{4}$ Division of Translational Informatics, Exploratory Oncology Research and Clinical Trial Center, National Cancer Center
}

Correspondence to: Hideki Makinoshima at hmakinos@east.ncc.go.jp

URL: https://www.jove.com/video/59551

DOI: doi:10.3791/59551

Keywords: Cancer Research, Issue 148, Metabolome, cancer cells, capillary electrophoresis-mass spectrometry (CE-MS), Glucose-6-phosphate dehydrogenase (G6PD), oxidative stress, diamide

Date Published: 6/9/2019

Citation: Maruyama, A., Kami, K., Sasaki, K., Sato, H., Sato, Y., Tsuchihara, K., Makinoshima, H. Extraction of Aqueous Metabolites from Cultured Adherent Cells for Metabolomic Analysis by Capillary Electrophoresis-Mass Spectrometry. J. Vis. Exp. (148), e59551, doi:10.3791/59551 (2019).

\section{Abstract}

Metabolomic analysis is a promising omics approach to not only understand the specific metabolic regulation in cancer cells compared to normal cells but also to identify biomarkers for early-stage cancer detection and prediction of chemotherapy response in cancer patients. Preparation of uniform samples for metabolomic analysis is a critical issue that remains to be addressed. Here, we present an easy and reliable protocol for extracting aqueous metabolites from cultured adherent cells for metabolomic analysis using capillary electrophoresis-mass spectrometry (CEMS). Aqueous metabolites from cultured cells are analyzed by culturing and washing cells, treating cells with methanol, extracting metabolites, and removing proteins and macromolecules with spin columns for CE-MS analysis. Representative results using lung cancer cell lines treated with diamide, an oxidative reagent, illustrate the clearly observable metabolic shift of cells under oxidative stress. This article would be especially valuable to students and investigators involved in metabolomics research, who are new to harvesting metabolites from cell lines for analysis by CE-MS.

\section{Video Link}

The video component of this article can be found at https://www.jove.com/video/59551/

\section{Introduction}

Otto Warburg observed that cancer cells acquire the unusual ability to take up glucose and ferment it to produce lactate in the presence of adequate oxygen - a phenomenon termed as Warburg effect or aerobic glycolysis ${ }^{1,2}$. Mitochondrial respiration defects are speculated as the underlying basis for aerobic glycolysis in cancer cells ${ }^{3}$. Indeed, the Warburg effect is the basis for tumor imaging by fluorodeoxyglucose (FDG)positron emission tomography (PET), which is widely used in clinical practice ${ }^{4,5}$. A high rate of aerobic glycolysis is considered a key feature of cancer and has been recently adopted as one of the well-known "hallmarks of cancer," as described by D. Hanahan and B. Weinberg ${ }^{6}$. Somatic mutations in oncogenes and tumor suppressor genes-such as HRAS/KRAS/NRAS, EGFR, BRAF, MYC, TP53, isocitrate dehydrogenase (IDH), and fumarate hydratase $(F H)$-have been linked to specific metabolic changes in cancer cells, believed to be a result of the Warburg effect ${ }^{7}$.

Metabolomic analysis is a promising approach not only to understand metabolic regulation in cancer cells but also to identify early-stage cancer biomarkers and chemotherapy response prediction. Following treatment of sensitive or resistant cancer cells with anticancer compounds, tracking of their metabolic responses facilitates identification of metabolic biomarkers to predict efficacy of specific anticancer therapies in cancer patients $^{8,9,10,11}$. In this article, cancer cell lines derived from a lung adenocarcinoma with an EGFR mutation treated with diamide-which causes oxidative stress-were used as models for metabolomic analysis. The advantage of this analytical method using capillary electrophoresis-mass spectrometry (CE-MS) is its comprehensive measurement of charged metabolites with the mass range $\mathrm{m} / \mathrm{z} 50-1000^{12,13}$. The purpose of this article is to provide novices a detailed stepwise visual protocol for preparation of aqueous metabolites from cultured cancer cells and subsequent metabolomic analysis, particularly by CE-MS

\section{Cell culture on day 1}

NOTE: Each sample for metabolite extraction should be prepared from a single $100 \mathrm{~mm}$ tissue culture dish that is moderately but not fully confluent (containing approximately 2-5 million cells). Calculate the number of dishes needed for the assay and prepare them accordingly. 
1. Culture $\mathrm{HCC} 827$ and PC-9 cells in $5 \% \mathrm{CO}_{2}$ at $37^{\circ} \mathrm{C}$ in RPMI- 1640 medium supplemented with $10 \%$ fetal bovine serum (FBS).

2. Aspirate the cell culture media from the $100 \mathrm{~mm}$ culture dishes.

3. Wash cells on each dish using $2 \mathrm{~mL}$ of phosphate buffered saline (PBS) solution without calcium and magnesium. Gently rock each dish so that the PBS solution completely covers the surface of the dish.

4. Aspirate the wash buffer from the culture dishes.

5. Warm $0.25 \%$ trypsin-EDTA solution to $37^{\circ} \mathrm{C}$ and add $2 \mathrm{~mL}$ of trypsin-EDTA solution with a $5 \mathrm{~mL}$ serological pipette. Gently rock each dish so that the trypsin completely covers the surface of the dish.

6. Incubate the culture dishes at $37^{\circ} \mathrm{C}$ for approximately $5 \mathrm{~min}$.

7. Add $4 \mathrm{~mL}$ of pre-warmed complete growth medium per dish. Resuspend the cells in medium by gently pipetting several times.

8. Transfer each cell suspension to a separate $15 \mathrm{~mL}$ conical tube and centrifuge at $800 \times g$ for $5 \mathrm{~min}$.

9. Resuspend each cell pellet in $2 \mathrm{~mL}$ of pre-warmed complete growth medium.

10. Determine the total number and percent viability of the cells using the automated cell counter and $0.4 \%$ trypan blue solution.

1. Mix $10 \mu \mathrm{L}$ of cell suspension and $10 \mu \mathrm{L}$ of $0.4 \%$ trypan blue solution.

2. Load $10 \mu \mathrm{L}$ of sample into the cell counting chamber slide through capillary action.

3. Insert the chamber slide into the automated cell counter. The transmitted light automatically illuminates and the instrument auto focuses on the cell.

4. Press the capture button to capture the image and display the results.

5. If necessary, add further growth medium to obtain the desired cell concentration.

11. Seed approximately 1-2.5 million cells per $100 \mathrm{~mm}$ cell culture dish.

NOTE: Metabolite concentrations determined by CE-MS analysis will be normalized based on the number of viable cells. For the purpose of cell counting, it is necessary to prepare at least one extra seeded culture dish for each group.

12. Incubate the culture dishes in $5 \% \mathrm{CO}_{2}$ at $37^{\circ} \mathrm{C}$ for $18 \mathrm{~h}$.

\section{Preparation of reagents}

1. Dilute a commercial internal standard solution including L-methionine sulfone and $d$-camphor-10-sulfonic acid 1000-fold in ultrapure water. NOTE: For fewer than 80 samples, simply mix $50 \mu \mathrm{L}$ of the Internal Standard Solution 1 and $45 \mathrm{~mL}$ of ultrapure water in a $50 \mathrm{~mL}$ volumetric flask, then bring the solution up to $50 \mathrm{~mL}$ with ultrapure water.

2. Prepare a $0.05 \mathrm{~g} / \mathrm{mL}$ mannitol solution in ultrapure water as wash buffer. NOTE: For fewer than 30 samples, simply dissolve $25 \mathrm{~g}$ of mannitol in $500 \mathrm{~mL}$ of ultrapure water. Approximately $15 \mathrm{~mL}$ of wash buffer is required per $100 \mathrm{~mm}$ culture dish, so prepare a sufficient volume of wash buffer according to the number of samples.

\section{Pre-washing centrifugal filter units}

1. Pipette $250 \mu \mathrm{L}$ of ultrapure water into the filter cup of each centrifugal filter unit (see the Table of Materials). NOTE: Two filter units per sample are required.

2. Cap the filter units tightly and centrifuge at $9,100 \times g$ at $4{ }^{\circ} \mathrm{C}$ for $5 \mathrm{~min}$.

3. Check the volume of each filtrate-if significant filtrate has accumulated during the first short spin, the filter unit may be defective. In this case, discard the filter unit and use a new filter unit instead.

4. Close the lids of the filter units tightly and centrifuge again at $9,100 \times g$ at $4{ }^{\circ} \mathrm{C}$ for $30 \mathrm{~min}$.

5. Ensure that no ultrapure water remains in any of the filter cups; remove the filtered ultrapure water in each collection tube with a pipette and discard.

NOTE: Do not try to remove residual water in a filter cup with a pipette as it may damage the filter.

6. Replace the filter cups into their collection tubes.

NOTE: Use the centrifugal filter units within an hour since the filters may become damaged upon drying.

\section{Cell culture on day 2}

1. Take out the $\mathbf{1 0 0} \mathbf{~ m m}$ culture dishes from the incubator.

NOTE: The recommended cell culture duration is $18 \mathrm{~h}$.

2. Aspirate the cell culture medium from each $100 \mathrm{~mm}$ culture dish.

3. Add $10 \mathrm{~mL}$ of cell culture medium that includes the appropriate concentrations of compounds or drugs to each dish, taking care to not disturb the cell layer.

NOTE: For demonstration purposes, we added $10 \mu \mathrm{L}$ of $250 \mathrm{mM}$ diamide dissolved in PBS (final concentration of $250 \mu \mathrm{M}$ ) in this experiment.

4. Incubate the culture dishes at $37^{\circ} \mathrm{C}$ for $30 \mathrm{~min}$ in the presence of diamide or PBS as a control.

5. Aspirate the cell culture medium from each $100 \mathrm{~mm}$ culture dish.

6. Wash cells by gently adding $2 \mathrm{~mL}$ of $5 \%$ mannitol solution to the edge of each dish, taking care to not to disturb the cell layer, then slightly tilt the dish.

NOTE: PBS or saline solution interferes with CE-MS-based metabolomic analysis and adversely affects measurement results, and thus should not be used as wash buffer.

7. Aspirate the wash buffer from each culture dish, then wash cells again by gently adding $10 \mathrm{~mL}$ of wash buffer per dish and slightly tilting the dish.

8. Completely aspirate the wash buffer from the edge of each culture dish.

NOTE: Aspirate as much wash buffer as possible, while paying attention to not aspirate the cells. Residual mannitol may interfere with CEMS analysis; aspiration of cells will decrease the number of cells and thus become a source of error in data normalization. 


\section{Extraction of metabolites from cultured cells}

1. Add $800 \mu \mathrm{L}$ of $99.7 \%$ methanol per culture dish. Gently rock each culture dish back and forth to cover its entire surface. Leave the dishes at room temperature for $30 \mathrm{~s}$.

2. Slowly add $550 \mu \mathrm{L}$ of the diluted internal standard solution per dish by immersing the tip of the pipette into the methanol and gently pipetting up and down several times.

3. Gently rock each culture dish back and forth to cover its entire surface.

4. Leave the dishes at room temperature for $30 \mathrm{~s}$.

\section{Ultrafiltration of cell extracts}

1. Transfer the extracted solution from each culture dish to a separate $1.5 \mathrm{~mL}$ microcentrifuge tube.

2. Centrifuge the tubes at $2,300 \times g$ at $4{ }^{\circ} \mathrm{C}$ for $5 \mathrm{~min}$.

3. Transfer $350 \mu \mathrm{L}$ of each supernatant into two centrifugal filter units per sample. NOTE: From each culture dish, a total of $700 \mu \mathrm{L}$ of the extracted solution is transferred into two filter tubes (350 $\mu \mathrm{L} / \mathrm{tube})$.

4. Centrifuge the filter tubes at $9,100 \times g$ at $4{ }^{\circ} \mathrm{C}$ for approximately $2 \mathrm{~h}$ until no liquid remains in the filter cups.

5. Remove the filter cups and tightly close the lids of the collection tubes.

\section{Sample evaporation}

1. Prepare a centrifugal evaporator-typically, this consists of an evaporator, a cold trap, and a vacuum pump.

2. Place the collection tubes in the centrifugal evaporator. NOTE: Leave the lids of the tubes open.

3. Evaporate the extracted sample solutions under vacuum conditions at room temperature. NOTE: Typical configurations for the number of rotations and pressure are 1,500 rpm and 1,000 Pa, respectively, and it usually takes approximately $3 \mathrm{~h}$ to completely evaporate the samples.

4. Confirm that no liquid remains in any of the collection tubes and close the lids of the tubes tightly.

5. Store the collection tubes in an ultra-low temperature $\left(-80^{\circ} \mathrm{C}\right)$ deep freezer until metabolomic analysis.

\section{Metabolomic analysis by CE-MS}

1. Resuspend the filtrate in $50 \mu \mathrm{L}$ of ultrapure water immediately before CE-MS analysis.

2. Perform CE-MS analysis by methods described previously ${ }^{12,13}$ using capillary electrophoresis system and time-of-flight mass spectrometer system equipped with an isocratic pump, a CE-MS adapter, and a CE-ESI-MS sprayer.

NOTE: Both systems can be controlled by the software of the system vendors and are connected by a fused silica capillary ( $50 \mu \mathrm{m}$ internal diameter $\times 80 \mathrm{~cm}$ total length).

1. Set up instruments and sample vials, prepare the capillary with a capillary cassette, replenish sheath liquids and appropriate electrophoresis buffers depending on anion or cation analysis mode, and then apply voltages. NOTE: Instrumentation and analytical conditions are described in detail elsewhere ${ }^{12,13}$.

2. Open the software and prepare a worklist containing data acquisition methods and sample information.

3. Start a test run and check the data such as the signal intensity and the peak shape of internal standards and the peak resolution of other standard compounds.

4. Fine-tune the analytical conditions if necessary.

5. Inject the sample solutions at $50 \mathrm{mbar}$ for $3 \mathrm{~s}$ and a voltage of $30 \mathrm{kV}$.

NOTE: CE-MS was conducted either in the positive or negative ion mode. Set the spectrometer to scan the mass range $\mathrm{m} / \mathrm{z} 50-1000$. The capillary voltage was set at $4 \mathrm{kV}$; the flow rate of nitrogen gas (heater temperature $300^{\circ} \mathrm{C}$ ). For positive mode, the fragmentor, skimmer and OCT RFV voltage were set at 75,50 and $125 \mathrm{~V}$, respectively. For negative ion mode, the fragmentor, skimmer, and OCT RFV voltage was set at 100,50 , and $200 \mathrm{~V}$, respectively.

3. Analyze spectrum data.

1. Extract peaks from the mass spectral data using automatic integration software in order to obtain peak information including $\mathrm{m} / \mathrm{z}$, peak area, and migration time (MT).

NOTE: The method is described in detail elsewhere ${ }^{14}$

2. Exclude signal peaks corresponding to isotopomers, adduct ions, and other product ions of known metabolites.

3. Annotate remaining peaks with information from the HMT metabolite database according to $\mathrm{m} / \mathrm{z}$ values and MTs

4. Normalize areas of the annotated peaks to internal standard levels and numbers of cells per sample.

5. Evaluate the concentration of each metabolite in the cultured cells ( $\mathrm{pmol} / 10^{6} \mathrm{cells}$ ) using standard curves prepared for each metabolite.

6. Use the quantified metabolite concentrations for subsequent statistical analyses and biological interpretations ${ }^{14}$. 


\section{Representative Results}

Since metabolite concentrations in cancer cells (pmol/10 $10^{6}$ cells) are normalized to the number of viable cells, experimental conditions should be set up with care so as to minimize variation in the number of viable cells between conditions. For example, diamide treatment was at a relatively high concentration $(250 \mu \mathrm{m})$ but for a short time to allow all the cells to grow as equally as possible, thereby equalizing the number of viable cells analyzed. Under these experimental conditions, HCC827 and PC-9 cells grew equally for $3 \mathrm{~h}$ (Figure 1). CE-MS analysis of diamide-treated cells compared with PBS-treated (control) cells revealed 175 and 150 differential metabolites in HCC827 and PC-9 cells, respectively. Among these, several intermediates in the pentose phosphate pathway (PPP) and in upper glycolysis were significantly higher in the diamide-treated conditions in both cell lines, whereas a few tricarboxylic acid (TCA) cycle intermediates were lower in the treated conditions (Figure 2 and Figure 3 ).

The PPP generates reducing equivalents in the form of reduced nicotinamide adenine dinucleotide phosphate (NADPH), which is used for redox homeostasis maintenance and fatty acid biosynthesis ${ }^{15}$. Following diamide treatment, the level of gluconic acid-an oxidized glucose -increased 12-fold in HCC827 cells and 10-fold in PC-9 cells; similarly, following diamide treatment, the level of glucose 6-phosphate (G6P) -a phosphorylated glucose and the first hexokinase-catalyzed glycolysis product-also increased 6.3- and 3.5-fold in HCC827 and PC-9 cells, respectively (Figure 4). In addition, following diamide treatment, the levels of 6-phosphogluconate (6PG) -the first intermediate in PPP —dramatically increased 89-fold in HCC827 cells and 231-fold in PC-9 cells compared to the levels seen in the PBS controls (Figure 4). In contrast, levels of other glycolytic intermediates, such as fructose 6-phosphate (F6P) and fructose 1,6-bisphosphate (F1,6P), did not change in the diamide experimental condition (Figure 4). Total nicotinamide adenine dinucleotide phosphate (NADP ${ }^{+}$) levels were nearly equivalent between diamide treatment and PBS control conditions (Figure 4), suggesting that glucose was mainly catabolized via the PPP.

A

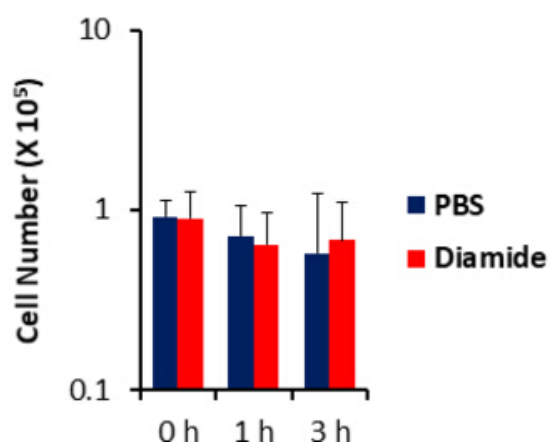

B

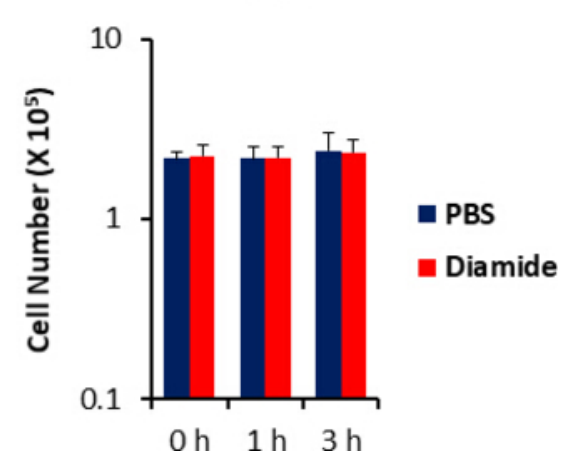

Figure 1. Unchanged cell numbers upon diamide treatment. Cell growth responses to $250 \mu \mathrm{m}$ of diamide were measured using trypan blue staining. Cell numbers of (A) HCC827 and (B) PC-9 cells treated with PBS (blue) or diamide (red; $250 \mu \mathrm{m})$ for 1 or $3 \mathrm{~h}$ are shown. Data are shown as the mean $\pm \mathrm{SD}(n=6)$. Please click here to view a larger version of this figure. 
A

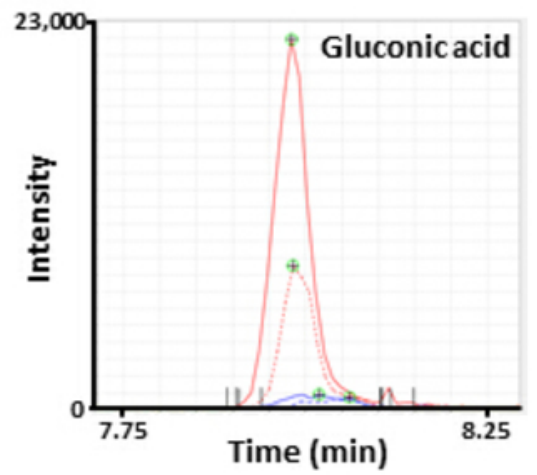

C

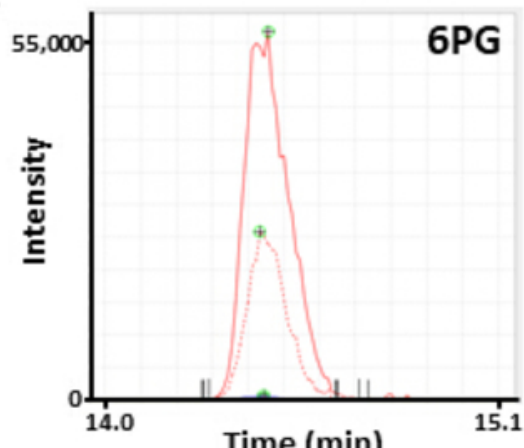

B

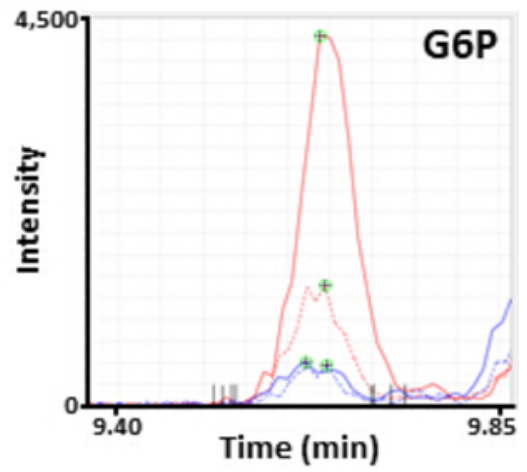

D

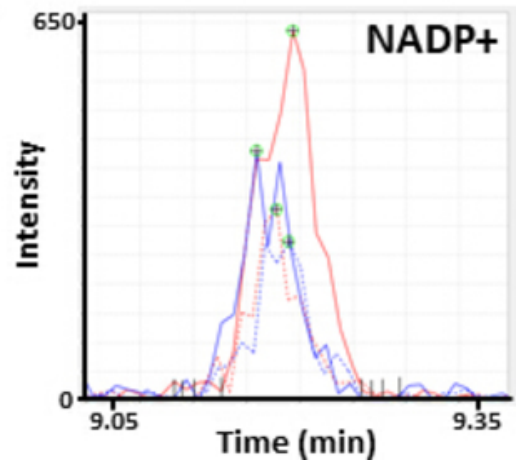

Figure 2. Representative MS peaks of metabolites. Electropherograms annotated as (A) gluconic acid, (B) glucose 6-phosphate (G6P), (C) 6phosphogluconate (6PG), and (D) nicotinamide adenine dinucleotide phosphate (NADP ${ }^{+}$) obtained by CE-MS analysis. Each line indicates the cell line (solid, HCC827; dotted, PC-9) and treatment (blue, PBS; red, diamide) used. Please click here to view a larger version of this figure. 


\section{A: HCC827}

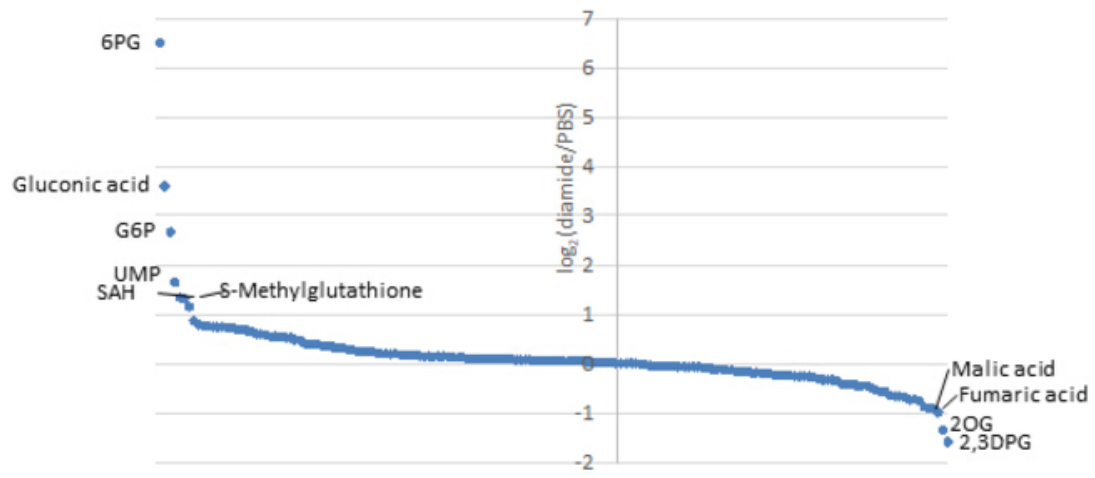

\section{B: PC-9}

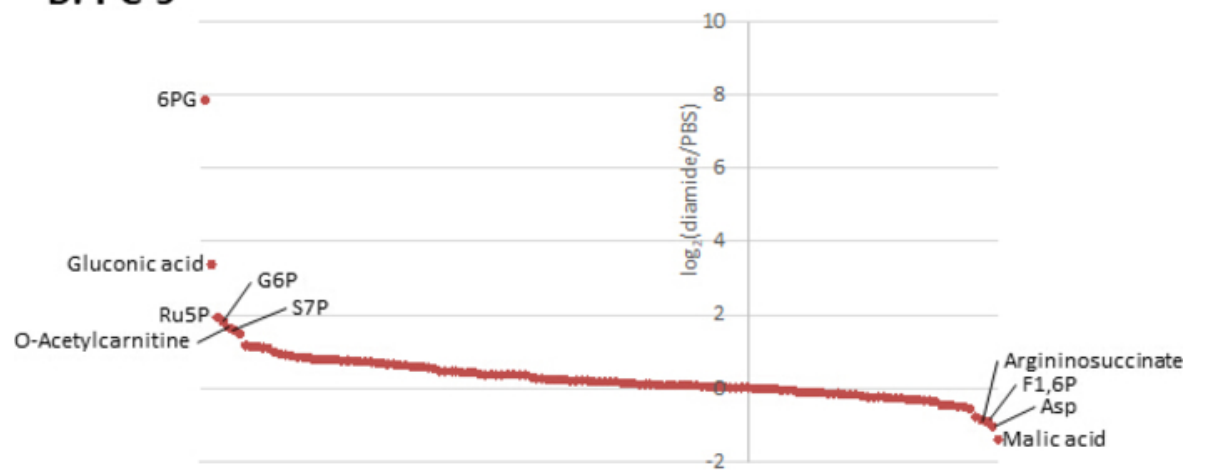

Figure 3. Metabolome profiles of intracellular metabolites. Fold changes of metabolites in (A) HCC 827 and (B) PC-9 cells treated with diamide are shown as $\log _{2}$ (diamide/PBS). In total, 175 and 150 metabolites were annotated in HCC827 and PC-9 cells, respectively. Please click here to view a larger version of this figure. 


\section{Gluconic acid}
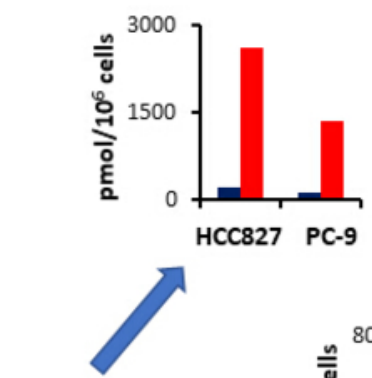

Glucose

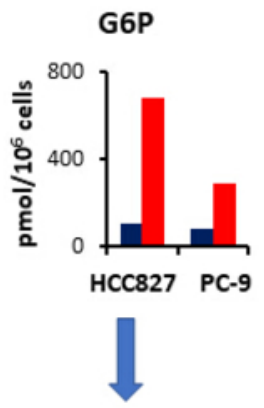

F6P

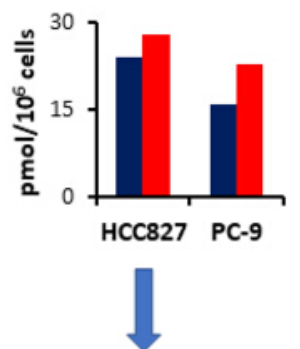

\section{Glycolysis}

Figure 4. Up-regulation of PPP upon diamide treatment. Intracellular concentrations (pmol/10 6 cells) of key metabolites involved in glycolysis and the pentose phosphate pathway $(P P P)$ after treatment with diamide are shown. Metabolites were extracted from HCC827 and PC-9 cells treated with PBS (b/ue) or diamide (red, $250 \mu \mathrm{m}$ ) for $30 \mathrm{~min}$. Representative metabolites such as gluconic acid, glucose 6-phosphate (G6P), fructose 6-phosphate (F6P), 6-phosphogluconate (6PG), and nicotinamide adenine dinucleotide phosphate (NADP ${ }^{+}$) are shown. Please click here to view a larger version of this figure.

\section{Discussion}

Here, we describe a widely accessible methodology to prepare metabolites from cultured cancer cells for CE-MS-based metabolomic analysis. One of the most critical points in this protocol is the proper preparation of cancer cells, because measured metabolite concentrations are normalized to the number of viable cells. For accurate estimation of cell number, it is necessary to prepare at least one additional culture dish per experimental group to count the number of viable cells in parallel with the extraction of metabolites for metabolomic analysis. In addition, the same number of cells should be seeded in each dish for the replicates and in the dish for counting; in the future, this would be aided by a quick and stressor-free (e.g., trypsin-free) cell counting protocol that allows the same dish to be used for both counting viable cells and extracting metabolites. Care should be taken during washes so that cells do not detach from the surface of the dishes. Severe cytotoxicity tests and other experiments that reduce cell adhesion may be unsuitable for this extraction protocol due to potential loss of cells during the washing procedure.

It is important to use a $5 \%$ mannitol solution as the wash buffer for extracting metabolites from cultured cells for CE-MS-based metabolomic analysis, because salt-based buffers, such as PBS, interfere with metabolomic analysis and adversely affect measurement.

Two or three dishes can be combined as a single sample by individually extracting metabolites from each dish and then pooling samples; however, combining multiple dishes often increases residual mannitol in the extracted metabolite solution. This may also interfere with metabolomic analysis by CE-MS. Hence, it is recommended to not use multiple dishes or wells as a single sample.

This metabolomic analysis method using CE-MS has been developed for comprehensive measurement of charged molecules with molecular weights between 50 and $1000 \mathrm{Da}$; thus, this protocol is optimized for extraction of aqueous, low molecular weight compounds. Therefore, this protocol is not suitable for extracting hydrophobic metabolites such as lipids or macromolecules such as proteins and nucleic acids. Since there is an increasing demand for comprehensive lipid analyses or lipidomics of cultured cell samples, the development of an easy and effective protocol for simultaneous extraction of both hydrophilic and hydrophobic metabolites is needed.

The first step of metabolite extraction—aspirating medium and washing cells with mannitol—should be conducted as quickly as possible to minimize changes to the metabolic profile of the cells. Treatment of cells with methanol after washing with mannitol is assumed to denature proteins and thereby prevent enzymes from catalyzing further metabolic reactions. However, even after methanol treatment, non-enzymatic chemical reactions—such as redox reactions, some decarboxylation processes, and thiol linkages—may take place. As such, any concentrations 
of metabolites involved in these reactions measured by this protocol should be interpreted with caution. In contrast to the genome or transcriptome, the metabolome consists of molecules with a wide variety of chemical properties; hence, no single protocol can extract all metabolites without any loss or disturbance. For more accurate measurements of such highly reactive metabolites, a protocol specifically designed to extract certain groups of metabolites, which requires fractionations and derivatizations, should be consulted. The protocol presented here, however, describes a simple and quick extraction of aqueous metabolites from cultured cell samples for metabolomic analysis by CE-MS. In this paper we could not describe how to set up CE-MS in detail because the focus of the present manuscript is different, however, describing detailed steps to set up CE-MS may require a separate dedicated article.

\section{Disclosures}

The authors have nothing to disclose.

\section{Acknowledgments}

We thank all the members of the Shonai Regional Industry Promotion Center for their help. This work was supported in part by research funds from Yamagata Prefecture and Tsuruoka City, by the National Cancer Center Research and Development Fund [grant number 28-A-9], and by the Japan Society for the Promotion of Science (JSPS) KAKENHI [grant number 17K07189] to HM.

\section{References}

1. Lunt, S. Y., Vander Heiden, M. G. Aerobic glycolysis: meeting the metabolic requirements of cell proliferation. Annual Review Cell and Developmental Biology. 27, 441-464, (2011).

2. Soga, T. Cancer metabolism: key players in metabolic reprogramming. Cancer Science. 104 (3), 275-281, (2013).

3. Zong, W. X., Rabinowitz, J. D., White, E. Mitochondria and Cancer. Molecular Cell. 61 (5), 667-676, (2016).

4. Fukuda, H., et al. Experimental study for cancer diagnosis with positron-labeled fluorinated glucose analogs: [18F]-2-fluoro-2-deoxy-Dmannose: a new tracer for cancer detection. European Journal of Nuclear Meddicine and Molecular Imaging. 7 (7), 294-297, (1982).

5. Miles, K. A., Williams, R. E. Warburg revisited: imaging tumour blood flow and metabolism. Cancer Imaging. 8, 81-86, (2008).

6. Hanahan, D., Weinberg, R. A. Hallmarks of cancer: the next generation. Cell. 144 (5), 646-674, (2011).

7. Levine, A. J., Puzio-Kuter, A. M. The control of the metabolic switch in cancers by oncogenes and tumor suppressor genes. Science. $\mathbf{3 3 0}$ (6009), 1340-1344, (2010).

8. Makinoshima, H., et al. Epidermal growth factor receptor (EGFR) signaling regulates global metabolic pathways in EGFR-mutated lung adenocarcinoma. The Journal of Biological Chemistry. 289 (30), 20813-20823, (2014).

9. Makinoshima, H., et al. Signaling through the Phosphatidylinositol 3-Kinase (PI3K)/Mammalian Target of Rapamycin (mTOR) Axis Is Responsible for Aerobic Glycolysis mediated by Glucose Transporter in Epidermal Growth Factor Receptor (EGFR)-mutated Lung Adenocarcinoma. The Journal of Biological Chemistry. 290 (28), 17495-17504, (2015).

10. Makinoshima, $\mathrm{H}$., et al. Metabolic Determinants of Sensitivity to Phosphatidylinositol 3-Kinase Pathway Inhibitor in Small-Cell Lung Carcinoma. Cancer Research. 78 (9), 2179-2190, (2018).

11. Sato, Y., et al. Metabolic Characterization of Antifolate Responsiveness and Non-responsiveness in Malignant Pleural Mesothelioma Cells. Frontiers in Pharmacology. 9, 1129, (2018).

12. Ohashi, Y., et al. Depiction of metabolome changes in histidine-starved Escherichia coli by CE-TOFMS. Molecular BioSystems. 4 (2), 135-147, (2008).

13. Ooga, T., et al. Metabolomic anatomy of an animal model revealing homeostatic imbalances in dyslipidaemia. Molecular BioSystems. 7 (4), 1217-1223, (2011)

14. Sugimoto, M., Wong, D. T., Hirayama, A., Soga, T., Tomita, M. Capillary electrophoresis mass spectrometry-based saliva metabolomics identified oral, breast and pancreatic cancer-specific profiles. Metabolomics. 6 (1), 78-95, (2010).

15. Patra, K. C., Hay, N. The pentose phosphate pathway and cancer. Trends in Biochemical Sciences. 39 (8), 347-354, (2014). 